\title{
Pathogenesis and transmission of avian influenza A (H7N9) virus in ferrets and mice
}

\author{
Jessica A. Belser ${ }^{1}$, Kortney M. Gustin ${ }^{1}$, Melissa B. Pearce ${ }^{1}$, Taronna R. Maines ${ }^{1}$, Hui Zeng ${ }^{1}$, Claudia Pappas ${ }^{1}$, Xiangjie Sun ${ }^{1}$, \\ Paul J. Carney ${ }^{1}$, Julie M. Villanueva ${ }^{1}$, James Stevens ${ }^{1}$, Jacqueline M. Katz ${ }^{1}$ \& Terrence M. Tumpey ${ }^{1}$
}

On 29 March 2013, the Chinese Center for Disease Control and Prevention confirmed the first reported case of human infection with an avian influenza $A(H 7 N 9)$ virus ${ }^{1}$. The recent human infections with $\mathrm{H} 7 \mathrm{~N} 9$ virus, totalling over 130 cases with 39 fatalities to date, have been characterized by severe pulmonary disease and acute respiratory distress syndrome (ARDS) ${ }^{2}$. This is concerning because $\mathrm{H} 7$ viruses have typically been associated with ocular disease in humans, rather than severe respiratory disease ${ }^{3}$. This recent outbreak underscores the need to better understand the pathogenesis and transmission of these viruses in mammals. Here we assess the ability of $A /$ Anhui/1/2013 and A/Shanghai/1/2013 (H7N9) viruses, isolated from fatal human cases, to cause disease in mice and ferrets and to transmit to naive animals. Both $\mathrm{H} 7 \mathrm{~N} 9$ viruses replicated to higher titre in human airway epithelial cells and in the respiratory tract of ferrets compared to a seasonal $\mathrm{H} 3 \mathrm{~N} 2$ virus. Moreover, the H7N9 viruses showed greater infectivity and lethality in mice compared to genetically related H7N9 and H9N2 viruses. The $\mathrm{H} 7 \mathrm{~N} 9$ viruses were readily transmitted to naive ferrets through direct contact but, unlike the seasonal H3N2 virus, did not transmit readily by respiratory droplets. The lack of efficient respiratory droplet transmission was corroborated by low receptorbinding specificity for human-like $\alpha 2,6$-linked sialosides. Our results indicate that $\mathrm{H} 7 \mathrm{~N} 9$ viruses have the capacity for efficient replication in mammals and human airway cells and highlight the need for continued public health surveillance of this emerging virus.

Laboratory-confirmed H7N9 infections in humans have been reported in eight contiguous provinces in eastern China (Anhui, Fujian, Henan, Hunan, Jiangsu, Jiangxi, Shandong and Zhejiang), two municipalities (Beijing and Shanghai) and Taiwan to date ${ }^{4}$. Although historically this subtype resulted in mild illness among humans ${ }^{3}$, the majority of novel $\mathrm{A}(\mathrm{H} 7 \mathrm{~N} 9)$ cases have resulted in severe respiratory illness in adults, representing the largest occurrence of $\mathrm{H} 7$ virus infections in humans ${ }^{2}$. Unlike highly pathogenic avian influenza (HPAI) H5N1 viruses, which exhibit lethality in poultry and cause severe human disease, low pathogenic (LPAI) H7N9 viruses seem to be asymptomatic or cause mild disease in poultry and wild bird populations, possibly due to the presence of a single amino acid in the haemagglutinin (HA) cleavage site of this virus ${ }^{2,5}$. Similar to H5N1 viruses, human cases with $\mathrm{H} 7 \mathrm{~N} 9$ virus seem to result from direct contact with infected poultry, yet $>20 \%$ of confirmed H7N9 cases have reported no exposure to live animals ${ }^{6}$. Although sustained human-to-human transmission has not been observed with this virus to date, reports of family clusters of A(H7N9) infection suggest the potential for virus spread between close contacts ${ }^{6}$. Understanding the properties of $\mathrm{A}(\mathrm{H} 7 \mathrm{~N} 9)$ viruses which contribute to human disease and their capacity for human-to-human transmission is a critical requirement for guidance of public health responses. The ferret (Mustela putorius furo) is recognized as the best small mammalian model for the concurrent study of influenza virus pathogenicity and transmissibility, and was used here to assess the virulence of $\mathrm{A}(\mathrm{H} 7 \mathrm{~N} 9)$ viruses.
A/Anhui/1/13 (Anhui/1) virus was isolated from a throat-swab specimen collected from an adult female patient with known poultry exposure and who died from ARDS 6 days after illness onset. A/Shanghai/1/ 13 (Shanghai/1) virus was isolated from an adult male patient with no known poultry exposure who died from refractory hypoxemia 13 days after illness onset ${ }^{2}$. These $\mathrm{A}(\mathrm{H} 7 \mathrm{~N} 9)$ viruses from China possess several molecular markers previously associated with human adaptation, including the presence of E627K in the PB2 protein and Q226L in the 210-loop in the HA gene $e^{2}$. Glycan microarray analysis of these phylogenetically distinct viruses revealed that Shanghai/1 (bearing Q226 in the HA (H3 numbering)) largely binds to avian $\alpha 2,3$ receptor analogues (glycans 4 to 40) (Fig. 1a and Supplementary Table 1), whereas Anhui/ 1 virus (bearing L226), revealed a mixed $\alpha 2,3 / \alpha 2,6$ receptor preference (Fig. $1 \mathrm{~b}$ and Supplementary Table 1); the single Q226L amino substitution has long been recognized to modulate receptor-binding specificity ${ }^{7}$. In particular, Anhui/1 virus bound to human biantennary structures (glycans 43 to 47), as well as the longer, linear $\alpha 2,6$ sialylated tri- $N$-acetyl lactosamine (glycans 58-60), some of which have been detected in $N$ glycans of cultured human bronchial epithelial cells ${ }^{8}$. These findings are in accord with recent studies showing that Anhui/1 virus binds to human tracheal tissue sections, albeit at a lower intensity than other human-adapted viruses ${ }^{9,10}$.

Select contemporary North American and Eurasian H7 viruses associated with human infection have previously demonstrated the capacity to transmit between ferrets in a direct-contact model, but not by respiratory droplets in the absence of direct contact ${ }^{11}$. To characterize the transmissibility of $\mathrm{A}(\mathrm{H} 7 \mathrm{~N} 9)$ isolates, we intranasally inoculated ferrets with $10^{6}$ plaque-forming units (p.f.u.) of virus Approximately $24 \mathrm{~h}$ post-inoculation (p.i.), inoculated-contact pairs were established by placing a naive ferret in the same cage as an inoculated ferret (direct contact transmission), or in an adjacent cage with perforated side-walls (respiratory droplet transmission) ${ }^{12}$. Nasal washes were collected on alternate days from all animals and titred for the presence of infectious virus ${ }^{12}$. The $\mathrm{A}(\mathrm{H} 7 \mathrm{~N} 9)$ viruses were compared to a seasonal H3N2 virus, A/Texas/50/2012 (Texas/50), representative of $\mathrm{H} 3 \mathrm{~N} 2$ viruses circulating in the 2012-2013 northern hemisphere season ${ }^{13}$.

Ferrets inoculated with Anhui/1 or Shanghai/1 virus showed modest weight loss and transient inactivity, but generally did not exhibit signs of severe disease (Table 1 and Supplementary Fig. 1). However, two of eight Shanghai/1 virus-inoculated ferrets were euthanized due to severe lethargy (laboured breathing, tremors, unresponsiveness day 4 p.i.) or excessive weight loss (26.6\% drop from initial body weight day 12 p.i.) (Table 1 and Supplementary Fig. 1). A(H7N9) virus was detected at elevated titres in the nasal turbinates and trachea (Supplementary Fig. 2). However, unlike the seasonal H3N2 virus, $\mathrm{A}(\mathrm{H} 7 \mathrm{~N} 9)$ virus was detected in the lower respiratory tract (peak mean titre $>10^{5}$ p.f.u. per g lung tissue) (Supplementary Fig. 1) ${ }^{10,14}$. Infectious virus was detected in the brain and olfactory bulb of $1 / 3$ and $2 / 3$ ferrets inoculated with Anhui/ 1 and Shanghai/1 viruses, respectively (Supplementary Fig. 2); viraemia or

${ }^{1}$ Influenza Division, National Center for Immunization and Respiratory Diseases, Centers for Disease Control and Prevention, Atlanta, Georgia 30333, USA. 

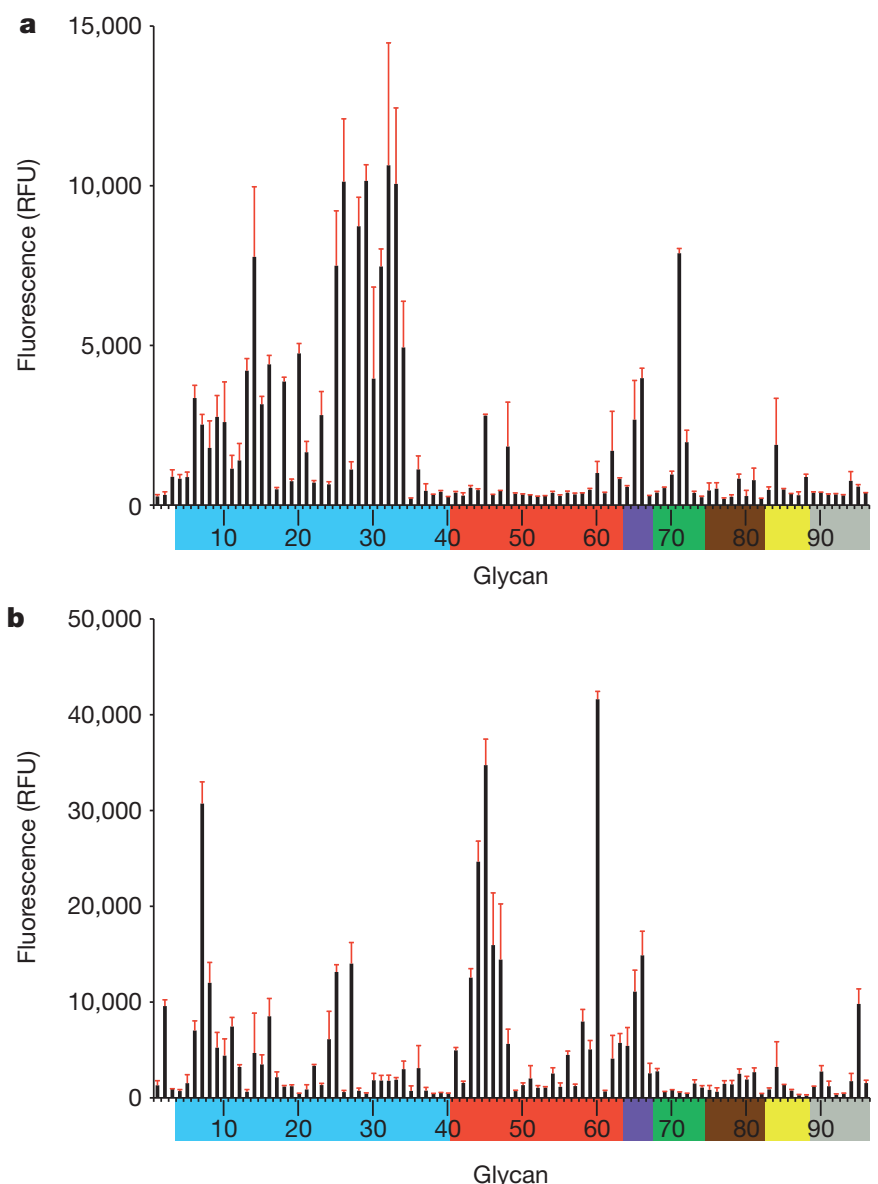

Figure $1 \mid$ Receptor specificity of A(H7N9) viruses. a, b, Glycan microarray analysis of Shanghai/1 (a) and Anhui/1 (b) viruses. Coloured bars highlight glycans that contain $\alpha 2,3$ sialosides (blue), $\alpha 2,6$ sialosides (red), $\alpha 2,6 / \alpha 2,3$ mixed sialosides (purple), $N$-glycolyl sialosides (green), $\alpha 2,8$ sialosides (brown), $\beta 2,6$ and 9-O-acetyl sialosides (yellow), and non-sialoside glycans (grey) and are expressed as relative fluorescence units (RFU). Error bars reflect the s.e.m. in the signal for six independent replicates on the array. Structures of each of the numbered glycans are found in Supplementary Table 1.

systemic spread of virus to the spleen, kidney or liver was not detected. Similar to the $2009 \mathrm{H} 1 \mathrm{~N} 1$ pandemic virus, gastrointestinal symptoms have been noted following $\mathrm{A}(\mathrm{H} 7 \mathrm{~N} 9)$ virus infection in humans ${ }^{5}$; rectal swabs were positive for virus detection in $7 / 11$ and 5/8 Anhui/1 and Shanghai/1 virus-inoculated ferrets, respectively, whereas $\mathrm{H} 3 \mathrm{~N} 2$ virus was not detected in this sample (Supplementary Fig. 3). Furthermore, intestinal tract samples were positive in 3/3 Shanghai/1 inoculated ferrets day 3 p.i. (Supplementary Fig. 2). In agreement with reports of lymphopenia among A(H7N9) virus-infected patients ${ }^{5}$, ferrets showed transient lymphopenia that was sustained through day 7 p.i., with greater reductions in circulating lymphocytes in $\mathrm{A}(\mathrm{H} 7 \mathrm{~N} 9)$ virus-infected ferrets compared to ferrets infected with H3N2 virus (Supplementary Table 2).
Ferrets inoculated with A(H7N9) virus shed high titres of virus in nasal washes as early as day 1 p.i., significantly higher than Texas/50 virus-infected ferrets at this time point $(P<0.05)$ (Table 1 and Supplementary Fig. 4). Mean titres in nasal washes were sustained at $>10^{5}$ p.f.u. in Anhui/ 1 and Shanghai/1 virus-infected ferrets through day 3 p.i. (Supplementary Fig. 4). Efficient direct contact transmission occurred by day 1 post-contact, with viral titres in nasal wash samples reaching comparable titres to inoculated ferrets by day 3 post-contact. Respiratory droplet transmission of Anhui/1 virus was observed in 2/6 ferrets, with detection of virus in contact ferret nasal washes delayed 2-4 days compared to seasonal H3N2 virus; Shanghai/1 virus was detected in $1 / 3$ ferrets in this model (Supplementary Fig. 4). These results are in accord with a recently published study demonstrating only limited transmissibility of A/Shanghai/2/13 virus by the airborne route $^{15}$. Sequence analysis of virus gene segments obtained from nasal wash samples of $\mathrm{A}(\mathrm{H} 7 \mathrm{~N} 9)$ respiratory droplet contact animals (total $n=3$ ) revealed no substantial amino acid changes. No sequence changes were observed from nasal washes obtained from the Shanghai/1 respiratory droplet contact ferret compared to Shanghai/1 sequences submitted to GISAID. Sequencing of nasal wash samples obtained on days 5-7 p.i. from Anhui/ 1 respiratory droplet contact ferrets revealed only one amino acid substitution in the HA protein at R140M (H3 numbering), not found among Anhui/ 1 inoculated ferrets. This position is located in the HA 140 loop region of HA1 and is not known to be associated with changes in $\mathrm{H7}$ HA receptor binding or influenza virus transmission. Although $\mathrm{A}(\mathrm{H} 7 \mathrm{~N} 9)$ viruses are not readily transmissible by respiratory droplets, the efficient virus transmission observed in the direct-contact model has not been observed with $\mathrm{H} 5 \mathrm{~N} 1$ viruses $^{12}$ and may indicate the capacity of an $\mathrm{A}(\mathrm{H} 7 \mathrm{~N} 9)$-like virus to acquire properties that would confer efficient airborne transmission.

Phylogenetic analysis showed that the H7N9 virus is a triple reassortant comprising an $\mathrm{HA}$ from an $\mathrm{H} 7 \mathrm{~N} 3$ virus, the neuraminidase (NA) of an H7N9 virus, and all six internal genes from an H9N2 virus ${ }^{2}$. To assess the relative contribution of these avian precursors to the pathogenicity of the $2013 \mathrm{H} 7 \mathrm{~N} 9$ viruses, we inoculated BALB/c mice intranasally with A/shoveler/Egypt/00215-NAMRU3/07 (shv/Egypt/07) ${ }^{16}$, an H7N9 virus with high surface glycoprotein amino acid sequence identity to Anhui/1, A/chicken/Vietnam/NCVD-1156/2011 (ck/VN/11), an $\mathrm{H} 9 \mathrm{~N} 2$ virus possessing internal genes with high genetic similarity to Anhui/1 virus, and the $2013 \mathrm{~A}(\mathrm{H} 7 \mathrm{~N} 9)$ viruses to determine virus replication, morbidity (as measured by weight loss), the $50 \%$ mouse infectious dose $\left(\mathrm{MID}_{50}\right)$, and the $50 \%$ mouse lethal dose $\left(\mathrm{LD}_{50}\right)$. $\mathrm{MID}_{50}$ titres were markedly low for all viruses, demonstrating high infectivity of the avian viruses in this model; typically human viruses do not replicate efficiently in mice without prior host adaptation ${ }^{17}$ (Table 2). Mice inoculated with Anhui/ 1 or Shanghai/ 1 virus showed severe morbidity ( $>20 \%$ weight loss) and a $\mathrm{LD}_{50}$ of $10^{3.3}-10^{3.4}$, a value comparable to the select group of HPAI H5N1 and H7N7 viruses which have a highpathogenicity phenotype in this model ${ }^{18,19}$. In contrast, the avian precursor viruses shv/Egypt/07 and ck/VN/11 caused moderate morbidity in mice, but did not display a lethal phenotype (Table 2). Collectively, lung titres in Anhui/1 and Shanghai/1 virus-inoculated mice were significantly higher than LPAI precursor viruses examined day 3 p.i. $(P<0.05)$; by day 6 p.i., all H7N9 viruses were detected at comparable

Table 1 | Clinical signs, virus replication, and transmission of H7N9 and H3N2 viruses in ferrets

\begin{tabular}{|c|c|c|c|c|c|c|c|c|c|}
\hline \multirow[b]{2}{*}{ Virus } & \multicolumn{5}{|c|}{ Inoculated animals } & \multicolumn{2}{|c|}{ Direct contact animals } & \multicolumn{2}{|c|}{ Respiratory droplet contact animals } \\
\hline & $\begin{array}{l}\text { Weight } \\
\text { loss } \dagger(\%)\end{array}$ & $\begin{array}{l}\text { Respiratory } \\
\text { symptoms: }\end{array}$ & Lethargy§ & Lethality॥ & $\begin{array}{c}\text { Nasal } \\
\text { wash titre }\end{array}$ & Virus detected & Seroconverted & Virus detected & Seroconverted \\
\hline Anhui/1 & 8.8 & $3 / 11$ & $1.1(7 / 11)$ & $0 / 11$ & $7.1(1)^{*}$ & $4 / 4$ & $4 / 4$ & $2 / 6$ & $2 / 6$ \\
\hline Shanghai/1 & 9.2 & $5 / 8$ & $1.3(7 / 8)$ & $2 / 8$ & $6.7(1)^{*}$ & $4 / 4$ & $4 / 4$ & $1 / 3$ & $1 / 3$ \\
\hline Texas/50 & 10.1 & $4 / 6$ & $1.0(2 / 6)$ & $0 / 6$ & $5.6(1)$ & $3 / 3$ & $3 / 3$ & $3 / 3$ & $3 / 3$ \\
\hline
\end{tabular}

$\dagger$ The percentage mean maximum weight loss observed during the first 10 days p.i.

* Number of ferrets that exhibited sneezing or nasal discharge during the first 10 days p.i.

$\S$ Relative inactivity index of ferrets during the first 10 days p.i. (number of ferrets with observed lethargy in parentheses).

॥Number of animals euthanized before the end of the 14 day experimental period because of reaching a clinical end point.

- Peak mean $\log _{10}$ (p.f.u. $\mathrm{ml}^{-1}$ ) nasal wash titre (day p.i. in parentheses). $* P<0.05$ compared with Texas $/ 50$ by one-way ANOVA 
Table 2 | Pathogenicity of $\mathrm{H7N} 9$ and related viruses in BALB/c mice

\begin{tabular}{lcccccc}
\hline Virus & Subtype & Weight loss $\dagger$ & $\begin{array}{c}\text { Lung } \\
\text { titre }(\mathrm{d} 3) !\end{array}$ & $\begin{array}{c}\text { Lung } \\
\text { titre }(\mathrm{d} 6) !\end{array}$ & $\mathrm{MID}_{50} \S$ & $\mathrm{LD}_{50} \S$ \\
\hline Anhui/1 & H7N9 & $22.2(7)$ & $7.2 \pm 0.6$ & $5.5 \pm 0.5$ & 0.25 & 3.4 \\
Shanghai/1 & H7N9 & $25.6(4)$ & $6.6 \pm 0.2$ & $5.6 \pm 0.2$ & 0.25 & 3.3 \\
shv/Egypt/07 & H7N9 & $16.5(3)$ & $4.4 \pm 0.2$ & $5.6 \pm 0.3$ & 2.25 & $>6$ \\
ck/VN/11 & H9N2 & $18.1(6)$ & $4.8 \pm 0.2$ & $3.1 \pm 0.1$ & 1.5 & $>6$ \\
\hline
\end{tabular}

$\dagger$ Maximum per cent weight loss (5 mice per group) after infection with $10^{6}$ p.f.u. of virus (day p.i. in parentheses).

\$ Average lung titres of three mice on indicated days p.i., expressed as $\log _{10}\left(\right.$ p.f.u. $\left.\mathrm{ml}^{-1}\right) \pm S D$.

$\S M I_{50}$ and $L D_{50}$ are expressed as the $\log _{10}$ p.f.u. $\mathrm{ml}^{-1}$ required to give one $\mathrm{MID}_{50}$ or one $\mathrm{LD}_{50}$.

titre, $>100$-fold higher than the H9N2 virus at this time point $(P<0.05)$. All viruses examined replicated to low titres in the nose day 3 and 6 p.i. (Supplementary Fig. 5), and systemic spread of virus to the brain was not detected.

Unlike past human infections with $\mathrm{H} 7$ viruses, conjunctivitis has not been reported among A(H7N9)-infected individuals ${ }^{5}$. Viral titres in ferret eye or conjunctiva samples were not frequently detected in $\mathrm{H} 7 \mathrm{~N} 9$ or $\mathrm{H} 3 \mathrm{~N} 2$ virus-infected animals, although conjunctival washes from $50 \%$ of Anhui/1 virus-infected ferrets were positive for virus (Supplementary Figs 2 and 3). Ocular inoculation of mice with shv/ Egypt, Anhui/1 or Shanghai/1 H7N9 viruses did not result in consistent virus replication in eye tissue (Supplementary Table 3). However, viral titres in the nose were detected days 3 and 6 p.i. following ocular inoculation with Anhui/ 1 and Shanghai/1 virus in mice with greater frequency compared to shv/Egypt/07 virus (Supplementary Table 3). These studies provide evidence that the 2013 A(H7N9) virus does not maintain the ocular tropism typical of $\mathrm{H} 7$ viruses, but is capable of using the eye as an entry portal to cause a productive respiratory infection.

The ability of Anhui/1 virus to form plaques in the presence or absence of the trypsin protease was assayed in MDCK cells by standard
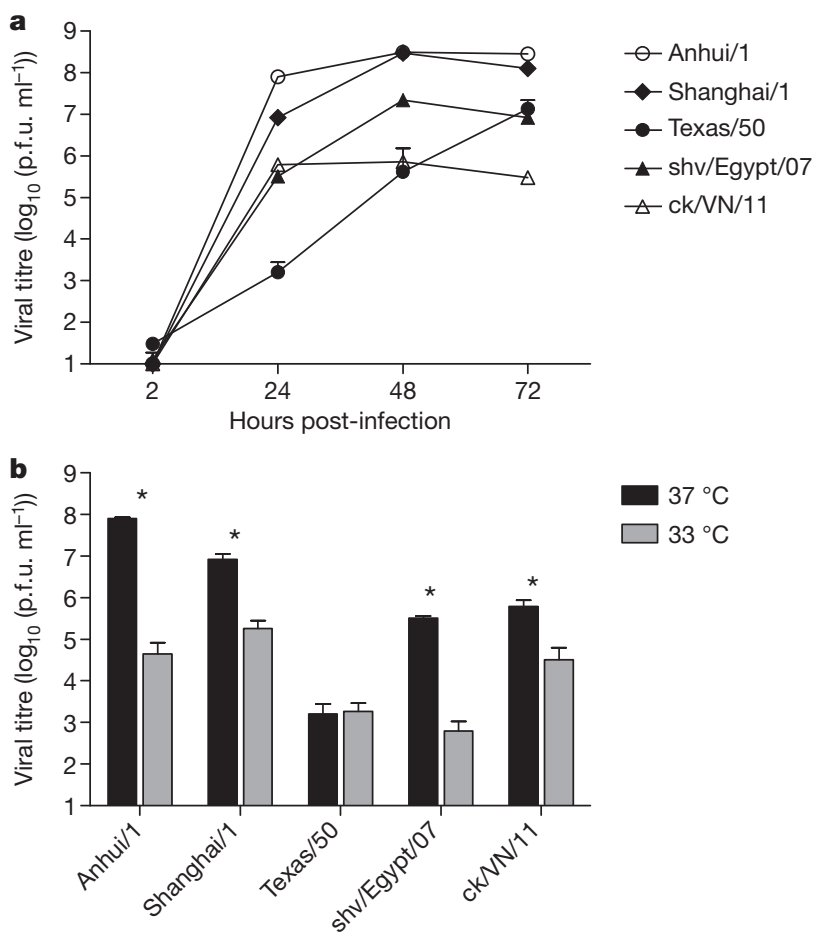

Figure 2 Replication kinetics of A(H7N9) influenza viruses in polarized human airway epithelial cells. a, Replication at $37^{\circ} \mathrm{C} ; \mathbf{b}$, viral titre at $24 \mathrm{~h}$ postinfection. Calu-3 cells cultured for 1 week were inoculated with virus at an MOI of 0.01. Culture supernatants were collected at the times indicated and virus titres were determined by plaque assay. Titre values represent the average for three independent wells plus s.d. *indicates statistically significant difference between $37^{\circ} \mathrm{C}$ and $33^{\circ} \mathrm{C}$ for each virus, based on the Mann-Whitney test $(P<0.004)$ plaque methods ${ }^{20}$. Proteolytic cleavage of the HA molecule is a prerequisite for multi-cycle replication, and the ability of virus to replicate in the absence of trypsin is thought to be an important determinant of influenza virus pathogenicity in mammals ${ }^{21}$. Similar to the seasonal Texas/ 50 virus which requires an exogenous protease source for multicycle replication and plaque formation, the $\mathrm{A}(\mathrm{H} 7 \mathrm{~N} 9)$ virus failed to form visible plaques without the addition of trypsin (data not shown).

Because the airway epithelium is the primary site of influenza virus replication in humans, we investigated the $\mathrm{A}(\mathrm{H} 7 \mathrm{~N} 9)$ virus replication efficiency in Calu-3 cells, derived from human bronchial epithelium and grown on permeable cell culture membranes to resemble the in vivo airway epithelium ${ }^{20}$ (Fig. 2a). For all viruses tested, titres of progeny virus progressively increased during the first $48 \mathrm{~h}$ p.i. Anhui/ 1 virus showed a 20 - to 400 -fold increase in replication at $48 \mathrm{~h}$ p.i. when compared to the other virus subtypes tested in Calu-3 cells $(P<0.04)$. Notably, compared to the seasonal H3N2 virus, Anhui/1 virus showed an 80,000 -fold increase in replication at $24 \mathrm{~h}$ p.i.

As previous studies have suggested an association between the transmissibility of influenza viruses in ferrets and their ability to replicate efficiently at the lower temperature $\left(33^{\circ} \mathrm{C}\right)$, found in the environment of the mammalian upper airway ${ }^{22}$, we evaluated the replication kinetics of $\mathrm{A}(\mathrm{H} 7 \mathrm{~N} 9)$ virus in Calu-3 cells cultured at either $33^{\circ} \mathrm{C}$ or $37^{\circ} \mathrm{C}$. Whereas the seasonal Texas/50 virus replicated equally well at both temperatures, $\mathrm{A}(\mathrm{H} 7 \mathrm{~N} 9)$ viruses showed significantly reduced replication at $33^{\circ} \mathrm{C}$ compared with $37^{\circ} \mathrm{C}$ at $24 \mathrm{~h}$, characteristic of avian subtype viruses (Fig. $2 \mathrm{~b})^{21}$; comparable titres at either temperature were detected by $48 \mathrm{~h}$ for all viruses (data not shown). These data demonstrate that the $\mathrm{A}(\mathrm{H} 7 \mathrm{~N} 9)$ viruses replicate productively in polarized bronchial epithelial cells at $37^{\circ} \mathrm{C}$, but less efficiently at the lower temperature of the human proximal airways early after infection.

The recent cases of human infection with H7N9 viruses highlight the need to better understand the potential of these viruses to spread and cause disease in humans. Although the novel H7N9 influenza virus has caused severe illness and death among individuals in China, the initial epidemiologic findings suggest that there is no evidence of sustained human-to-human transmission of this virus. Our ferret transmission results are consistent with this observation and suggest that additional virus adaptation in mammals would be required to reach the high-transmissible phenotypes observed by the respiratory droplet route with pandemic and seasonal influenza A viruses ${ }^{10}$.

\section{METHODS SUMMARY}

Viruses and cells. H7N9 and H9N2 viruses were passaged in eggs; $\mathrm{H} 1 \mathrm{~N} 1$ virus was propagated in MDCK cells. Detailed culture conditions for Calu-3 cells are included in Methods. Virus titrations were performed in MDCK cells by standard plaque assay ${ }^{20}$.

Biosafety and biosecurity. Experiments were conducted in biosafety level 3 facilities with enhancements (BSL3+) in accordance with U.S. Federal and WHO guidelines. Features of the BSL3 + containment laboratories include a double door entrance, double door autoclave, HEPA filtration of intake and exhaust air, shower out room, and a liquid effluent sterilizer. Security is monitored through an automated access control system and entrance to the laboratory is restricted by guard stations, cameras, card-controlled doors and biometric readers. Use of powered air-purifying respirators in the laboratory and yearly training requirements are mandatory for all staff with access.

Glycan arrays. Microarray printing and influenza virus analyses were performed as previously described ${ }^{11}$; specific details on the glycans analysed are provided in Methods.

Animals. Six-to-eight week old female BALB/c mice (Jackson Laboratory) and six-to-seven month old influenza virus-seronegative male ferrets (Triple F Farms) were used in all experiments. All intranasally inoculated animals were administered $10^{6}$ p.f.u. of virus except for determination of mouse infectivity and lethality, where mice were administered serial 10 -fold dilutions of virus. Virus transmission in ferrets was measured by co-housing an inoculated and naive ferret (direct contact transmission) or by placing an inoculated and naive ferret in adjacent cages with a perforated side wall (respiratory droplet transmission) ${ }^{12}$. Transmission was determined by presence of virus in nasal washes and seroconversion 
of contact ferrets. Detailed procedures are included in Methods for pathotyping, transmission and tropism studies.

Full Methods and any associated references are available in the online version of the paper.

Received 18 May; accepted 20 June 2013.

Published online 10 July 2013.

1. WHO. Avian influenza A(H7N9) virus http://www.who.int/influenza/ human_animal_interface/influenza_h7n9/en/index.html (2013).

2. Gao, R. et al. Human infection with a novel avian-origin influenza A (H7N9) virus, N. Engl. J. Med. 368, 1888-1897 (2013).

3. Belser, J. A., Bridges, C. B., Katz, J. M. \& Tumpey, T. M. Past, present, and possible future human infection with influenza virus A subtype H7. Emerg. Infect. Dis. 15, 859-865 (2009).

4. CDC. Emergence of avian influenza $A(H 7 N 9)$ virus causing severe human illness China, February-April 2013. MMWR Morb. Mortal. Wkly. Rep. 62, 1-4 (2013).

5. Chen, Y. et al. Human infections with the emerging avian influenza A H7N9 virus from wet market poultry: clinical analysis and characterisation of viral genome. Lancet 381, 1916-1925 (2013).

6. Li, Q. etal. Preliminary report: epidemiology of the avian influenza A (H7N9) outbreak in China. N. Engl. J. Med. http://dx.doi.org/10.1056/NEJMoa1304617 (2013).

7. Rogers, G. N. et al. Single amino acid substitutions in influenza haemagglutinin change receptor binding specificity. Nature 304, 76-78 (1983).

8. Chandrasekaran, A. et al. Glycan topology determines human adaptation of avian H5N1 virus hemagglutinin. Nature Biotechnol. 26, 107-113 (2008).

9. Tharakaraman, K. et al. Glycan receptor binding of the influenza A virus H7N9 hemagglutinin. Cell 153, 1486-1493 (2013).

10. Maines, T. R. et al. Transmission and pathogenesis of swine-origin 2009 A(H1N1) influenza viruses in ferrets and mice. Science 325, 484-487 (2009).

11. Belser, J. A. et al. Contemporary North American influenza $\mathrm{H} 7$ viruses possess human receptor specificity: Implications for virus transmissibility. Proc. Natl Acad. Sci. USA 105, 7558-7563 (2008).

12. Maines, T. R. et al. Lack of transmission of H5N1 avian-human reassortant influenza viruses in a ferret model. Proc. Natl Acad. Sci. USA 103, 12121-12126 (2006).

13. WHO. Recommended composition of influenza virus vaccines for use in the 20132014 northern hemisphere influenza season http://www.who.int/influenza/ vaccines/virus/recommendations/201302_recommendation.pdf (2013).

14. Zitzow, L. A. et al. Pathogenesis of avian influenza $A(H 5 N 1)$ viruses in ferrets. J. Virol. 76, 4420-4429 (2002).

15. Zhu, H. et al. Infectivity, transmission, and pathology of human H7N9 influenza in ferrets and pigs. Science http://dx.doi.org/101126/science.1239844 (2013).
16. Gerloff, N. A. et al. A high diversity of Eurasian lineage low pathogenicity avian influenza A viruses circulate among wild birds sampled in Egypt. PLOS ONE (in the press).

17. Hartley, C. A., Reading, P. C., Ward, A. C. \& Anders, E. M. Changes in the hemagglutinin molecule of influenza type A (H3N2) virus associated with increased virulence for mice. Arch. Virol. 142, 75-88 (1997).

18. Maines, T. R. et al. Avian influenza (H5N1) viruses isolated from humans in Asia in 2004 exhibit increased virulence in mammals. J. Virol. 79, 11788-11800 (2005).

19. de Wit, E. et al. Protection of mice against lethal infection with highly pathogenic H7N7 influenza A virus by using a recombinant low-pathogenicity vaccine strain. J. Virol. 79, 12401-12407 (2005).

20. Zeng, H. et al. Highly pathogenic avian influenza $\mathrm{H} 5 \mathrm{~N} 1$ viruses elicit an attenuated type I interferon response in polarized human bronchial epithelial cells. J. Virol. 81, 12439-12449 (2007)

21. Zeng, H. et al. Tropism and infectivity of influenza virus, including highly pathogenic avian $\mathrm{H} 5 \mathrm{~N} 1$ virus, in ferret tracheal differentiated primary epithelial cell cultures. J. Virol. 87, 2597-2607 (2013).

22. Van Hoeven, N. et al. Pathogenesis of 1918 pandemic and H5N1 influenza virus infections in a guinea pig model: antiviral potential of exogenous alpha interferon to reduce virus shedding. J. Virol. 83, 2851-2861 (2009).

Supplementary Information is available in the online version of the paper.

Acknowledgements We thank the China CDC as part of the WHO Global Influenza Surveillance and Response System (GISRS) for facilitating access to viruses and A. Balish for preparation of viruses. The findings and conclusions in this report are those of the authors and do not necessarily reflect the views of the funding agency. Glycan microarray slides were produced under contract for the CDC using a glycan library generously provided by the Consortium for Functional Glycomics, funded by National Institute of General Medical Sciences Grant GM62116.

Author Contributions J.A.B., K.M.G., T.R.M., H.Z., X.S., P.J.C., J.S., J.M.K., and T.M.T. designed the experiments; J.A.B., K.M.G., M.B.P., T.R.M.,H.Z., C.P., X.S., P.J.C., J.S., and T.M.T. performed the experiments; J.A.B., K.M.G., T.R.M., H.Z., C.P., X.S., P.J.C., J.M.V., J.S. J.M.K., and T.M.T. analysed data; J.A.B., T.R.M., H.Z., C.P., P.J.C., J.S., and J.M.K., T.M.T. wrote the manuscript.

Author Information GISAID (http://platform.gisaid.org/) accession numbers are as follows for ck/VN/11 (PB2, EPI457480; PB1, EPI457481; PA, EPI457479; HA, EPI457483; NP, EPI457476; NA, EPI457482; MP, EPI457478; NS, EPI457477) and shv/Egypt/07 (PB2, EPI372407; PB1, EPI372408; PA, EPI372406; HA, EPI372410; NP, EPI372403; NA, EPI372409; MP, EPI372405; NS, EPI372404). Reprints and permissions information is available at www.nature.com/reprints. The authors declare no competing financial interests. Readers are welcome to comment on the online version of the paper. Correspondence and requests for materials should be addressed to T.M.T. (tft9@cdc.gov). 


\section{METHODS}

Viruses. All animal experiments were conducted under biosafety level 3 facilities with enhancements (BSL3+) in accordance with US Federal and World Health Organization guidelines. Biocontainment for the BSL3+ laboratories is based on the 5th Edition of Biosafety in Microbiological and Biomedical Laboratories. A/Anhui/1/13 (H7N9), A/Shanghai/1/13 (H7N9), A/shoveler/Egypt/ 00215-NAMRU3/07 (H7N9), A/chicken/Vietnam/NCVD-1156/2011 (H9N2) and A/Goose/Nebraska/17097-4/11 (H7N9) viruses were propagated in 10-dayold embryonated hens' eggs for $42 \mathrm{~h}$ at $37^{\circ} \mathrm{C}$ as previously described ${ }^{18,23}$. A/Texas/ 50/12 was propagated in Madin Darby canine kidney (MDCK) cells as previously described ${ }^{24}$.

Ferret experiments. All animal research was approved by the Centers for Disease Control and Prevention's Institutional Animal Care and Use Committee and conducted in an Association for Assessment and Accreditation of Laboratory Animal Care International-accredited animal facility. Male Fitch ferrets (Triple F Farms), 6 to 7 months of age and serologically negative by haemagglutination inhibition (HI) assay for currently circulating influenza viruses, were used in all experiments. A minimum number of animals were used to achieve reproducible results in each experiment per ethical guidelines; investigators were not blinded to groups. Ferrets were housed in cages within a Duo-Flow Bioclean mobile clean room (Lab Products) and randomly assigned to experimental groups. Temperatures were measured using a subcutaneous implantable temperature transponder (BioMedic Data Systems). Ferrets were inoculated intranasally with $10^{6}$ p.f.u. of virus in a $1 \mathrm{ml}$ volume diluted in PBS, then monitored daily for changes in body temperature, weight and clinical signs of infection. Lethargy was measured as described previously ${ }^{14}$. Any ferret that lost $>25 \%$ of its pre-inoculation body weight or showed neurological dysfunction was euthanized and submitted to post-mortem examination. Nasal washes, conjunctival washes and rectal swabs were collected on alternate days post-inoculation (p.i.) or post-contact, immediately frozen on dry ice and stored at $-70{ }^{\circ} \mathrm{C}$ until titration as previously described ${ }^{25}$. Tissue specimens collected for virus titration were immediately frozen on dry ice and stored at $-70{ }^{\circ} \mathrm{C}$ until titration as previously described ${ }^{18}$. Blood was collected from inoculated ferrets on indicated days p.i. in EDTA Vacutainer tubes (BD) and complete blood counts were quantified using a Hemavet HV950FS instrument per the manufacturer's instructions (Drew Scientific).

For transmission experiments, ferrets were inoculated as described above. Approximately $24 \mathrm{~h}$ after inoculation, a naive ferret was placed in the same cage as an inoculated animal (for assessment of virus transmission in the presence of direct contact) or in an adjacent cage with perforated side-walls (holes $1-5 \mathrm{~mm}$ in diameter, with cages spaced $\sim 3 \mathrm{~mm}$ apart) to allow for air exchange between cages in the absence of direct or indirect contact (for assessment of virus transmission by respiratory droplets). The term 'respiratory droplet transmission' refers to virus transmission in the absence of direct or indirect contact and does not imply an understanding of the size of droplets or aerosols involved in the transmission events. To prevent inadvertent transmission in direct contact or respiratory droplet experiments, investigators always handled the contact animals first, and decontaminated all surfaces which came into contact with each ferret before handling the next animal.

Whole-genome sequencing of nasal washes. RNA was extracted from nasal wash samples from the peak day of virus shedding presenting in the inoculated and respiratory droplet contact (2/6 for Anhui/ 1 ferrets, and 1/3 for Shanghai/ 1 virus experiment) ferrets and subjected to RT-PCR (PCR with reverse transcription) followed by DNA sequencing analysis. Obtained sequences obtained from all virus gene segments were compared with relevant virus sequences available from The Global Initiative on Sharing All Influenza Data (GISAID) database.

Mouse experiments. Female BALB/c mice 6 to 9 weeks old were anaesthetized with an intraperitoneal injection of $0.15 \mathrm{ml}$ of 2,2,2-tribromoethanol in tert-amyl alcohol (Avertin; Sigma-Aldrich) prior to virus inoculation. Intranasal inoculations were performed by instilling $50 \mu \mathrm{l}$ of virus diluted in PBS onto the nares of the animals. The $50 \%$ mouse infectious dose $\left(\mathrm{MID}_{50}\right.$, determined by virus detection in the lungs day 3 p.i) and $50 \%$ lethal dose $\left(\mathrm{LD}_{50}\right)$ were determined by inoculating groups of eight mice with serial 10 -fold dilutions of virus. Three mice per dilution were euthanized on day 3 p.i.; lungs were collected from each mouse, immediately frozen on dry ice, and stored at $-70^{\circ} \mathrm{C}$. Tissues were thawed, homogenized in $1 \mathrm{ml}$ cold PBS, and clarified by centrifugation prior to titration by standard plaque assay. Five mice per dilution were observed daily for 14 days for morbidity (as measured by weight loss) and mortality; any mouse which lost $>25 \%$ of its preinoculation weight was euthanized. $\mathrm{MID}_{50}$ and $\mathrm{LD}_{50}$ values were determined following ref. 26. To investigate the ability of each virus to spread systemically in mice, an additional three mice were euthanized at days 3 and 6 p.i. from mice inoculated with $10^{6}$ p.f.u. of each virus for collection of nose, lung and brain tissue, which were stored and processed as described above.

Ocular inoculations were performed by lightly scarifying the right eye of the mouse with a corneal trephine and instilling $5 \mu$ l of virus $\left(10^{6}\right.$ p.f.u. $)$ onto the surface of the eye and massaging the virus into the eye with the eyelid as previously described ${ }^{27}$. An additional three mice were inoculated and euthanized on days 3 and 6 p.i. for collection of eye, nose and lung tissue, which were stored and processed as described above.

Cell culture and viral replication. The human bronchial epithelial cell line Calu-3 (ATCC) was grown on membrane inserts as previously described ${ }^{20}$. Cells were grown to confluence in six-well plates for 1 week until the confluent monolayer reached a stable transepithelial resistance of $>1,000 \Omega \mathrm{cm}^{2}$. Virus was added to cells apically in serum-free medium at a multiplicity of infection (MOI) of 0.01 for one hour before washing. Plates were incubated at either $37^{\circ} \mathrm{C}$ or $33^{\circ} \mathrm{C}$ for the duration of the experiment as indicated. Aliquots of culture supernatant taken p.i. were immediately frozen at $-80^{\circ} \mathrm{C}$ until titration. All samples collected for replication kinetics were titrated for the presence of infectious virus by standard plaque assay in MDCK cells.

Serology. HI assays were performed with ferret sera collected 16-20 days after inoculation or exposure to infected animals using $0.5 \%$ turkey erythrocytes against homologous virus ${ }^{28}$

Cytokine and chemokine quantification. Clarified mouse lung tissue homogenates were analysed with the BioPlex protein array system (Bio-Rad), according to manufacturer's instructions. A minimum of three independent samples were collected and tested in duplicate for each condition.

Glycan microarray analysis. Microarray printing and influenza virus analyses have been described previously ${ }^{11}$. A/Anhui/1/2013 virus was analysed at a HA titre of 512 while A/Shanghai/1/2013 was analysed at an HA titre of 256 . Specific details on the glycans analysed in these studies are provided in Supplementary Table 1. Statistics. Statistical significance $(P<0.05)$ for murine and ferret studies was determined by one-way analysis of variance (ANOVA) with a Bonferroni posttest. Statistical significance for in vitro studies was determined using the MannWhitney and Student's $t$ tests. All in vitro studies were conducted with sufficient sample size for statistical analyses performed; indicated tests were chosen as appropriate for each data set to be analysed and all data meet the assumptions of the tests.

23. Belser, J. A. et al. Pathogenesis, transmissibility, and ocular tropism of a highly pathogenic avian influenza A (H7N3) virus associated with human conjunctivitis. J. Virol. 87, 5746-5754 (2013).

24. Tumpey, T. M. et al. Characterization of the reconstructed 1918 Spanish influenza pandemic virus. Science 310, 77-80 (2005).

25. Belser, J. A. et al. Influenza virus respiratory infection and transmission following ocular inoculation in ferrets. PLoS Pathog. 8, e1002569 (2012).

26. Reed, L. J. \& Muench, H. A simple method of estimating fifty per cent endpoints. Am. J. Hyg. 27, 493-497 (1938).

27. Belser, J. A., Wadford, D. A., Xu, J., Katz, J. M. \& Tumpey, T. M. Ocular infection of mice with influenza $A(H 7)$ viruses: a site of primary replication and spread to the respiratory tract. J. Virol. 83, 7075-7084 (2009).

28. Stephenson, I., Wood, J. M., Nicholson, K. G., Charlett, A. \& Zambon, M. C. Detection of anti-H5 responses in human sera by HI using horse erythrocytes following MF59-adjuvanted influenza A/Duck/Singapore/97 vaccine. Virus Res. 103, 91-95 (2004). 